\section{RNAi-mediated resistance to SMV and BYMV in transgenic tobacco}

\author{
Lo Thi Mai Thu ${ }^{1}$, Vi Thi Xuan Thuy ${ }^{1}$, Le Hoang Duc ${ }^{2}$, Le Van Son ${ }^{2}$, \\ Chu Hoang $\mathrm{Ha}^{2}$ and Chu Hoang $\mathrm{Mau}^{3 *}$
}

\begin{abstract}
Soybean mosaic virus (SMV) and bean yellow mosaic virus (BYMV) are two typical types of viruses that cause mosaic in soybean plants. Multiple viral infections at the same site can lead to $66 \%$ to $80 \%$ yield reduction. We have aimed to improve SMV and BYMV resistance in Vietnamese soybeans using gene transfer techniques under the mechanism of RNAi. In this study, we present newly generated transgenic tobacco plants carrying RNAi [CPi (SMV-BYMV)] resistance to the two types of viruses; $73.08 \%$ of transgenic tobacco lines proved to be fully resistant to SMV and BYMV. In addition, the number of virus copies in transgenic tobacco plants was reduced on average by more than $51 \%$ compared to the control plants (wild type). This promising result shows the potential of transerring the CPi (SMV-BYMV) structure in soybean to increase resistance of soybean to $S M V$ and BYMV and advance the aims of antiviral soybean breeding in Vietnam.
\end{abstract}

Key words: Antiviral soybean breeding, gene transfer, Glycine max, qRT-PCR, mosaic virus.

\section{INTRODUCTION}

Soybean mosaic virus (SMV) and Bean yellow mosaic virus (BYMV) belong to the Potyvirus genus, which causes mosaic in legume crops. SMV and BYMV can infect the same plant simultaneously and can be very difficult to distinguish. Multiple infections from different types of viruses can reduce soybean yields from $66 \%$ to $80 \%$ (Hartman et al. 1999). Currently, the main methods for reducing losses from these two viruses are still traditional preventive measures, such as selecting clean lines for seed banks, field sanitation, crop rotation, cleanup of diseased crops, pest and disease control, and so on. These methods are very time-consuming, inefficient, and unsustainable. An effective measure to prevent SMV and BYMV is to use virus-resistant cultivars. However, natural strains resistant to SMV and BYMV are very limited. Therefore, a new approach is to generate transgenic plants resistant to viruses. One promising approach is utilizing an RNA interference (RNAi) mechanism. RNAi is considered an effective technique to improve plant resistance against viruses. RNAi is a process of post-transcriptional gene silencing (PTGS) triggered by double-stranded RNA (dsRNA) (Baulcombe 2004). Numerous groups have successfully applied RNAi strategies in generating transgenic plants resistant to viruses (Asad et al. 2003, Kasai et al. 2012). Transgenic plants have slowed the accumulation of disease symptoms or reduced them. In 2007, using the RNAi approach, Bonfim et al. obtained a transgenic soybean line with high resistance to Bean golden mosaic virus (up to 93\%). Another study (Furutani et al. 2007) also tried to improve SMV resistance in soybean using genetic engineering techniques.
Crop Breeding and Applied Biotechnology 16: 213-218, 2016 Brazilian Society of Plant Breeding. Printed in Brazil http://dx.doi.org/10.1590/1984$70332016 v 16 n 3 a 32$

(1)

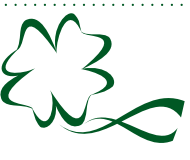

\title{
(70332016v16n3as2
}


We designed an RNAi-based construct to silence genes encoding a coat protein (CP) of SMV and BYMV, thus limiting viral infection. From conserved regions of CP coding sequences of SMV and BYMV, we cloned a 573 bp cDNA fragment, called CPi (SMV-BYMV), which is composed of a 294 bp fragment from the SMV CP gene and a 279 bp fragment from the BYMV CP gene. The CPi (SMV-BYMV) fragment was then cloned in sense- and antisense-orientation into the vector pK7GWIWG2 (Karimi et al. 2002) by using Gateway technology (Invitrogen). The final construct is called pK7GW-CPi (SMVBYMV), which we were able to transcribe in the cells to generate the hairpin RNA, thus inducing the RNAi mechanism to degrade the CP gene of SMV and BYMV. This was presented in our previous publication (Lo et al. 2014). In this study, we analyze resistance to SMV and BYMV in transgenic tobacco lines carrying the RNAi structure.

\section{MATERIAL AND METHODS}

\section{Materials}

Tobacco plants (Nicotiana tabacum) of the cultivar C9-1 were provided by the Vietnam Tobacco Economic Technical Institute. The SMV and BYMV viruses were provided by the Plant Protection Research Institute. The pK7GW-CPi (SMVBYMV) vector was constructed based on the Gateway principles, which were described by Karimi et al. (2002) and transformed into the Agrobacterium tumefaciens strain CV58 PGV 2660.

The Polymerase Chain Reaction (PCR) primers SMV-CPi-Fi/BYMV-CPi-Ri were used to check for the presence of the $\mathrm{CPi}$ gene in transgenic plants, and the CP gene fragment was amplified by using primers SMVqF/SMVqR for real-time PCR (RT-PCR) (Table 1).

\section{Methods}

Agrobacterium-mediated transformation via leaf infection and regeneration of tobacco plants was carried out as previously described by Topping (1998). Total DNA was isolated from young leaves based on the method of Saghai et al. (1984).

Transgenic tobacco plants were grown in a greenhouse for 2-3 weeks to acquire 5 true leaves (each about $10 \mathrm{~cm}$ in length), which were then infected by SMV and BYMV by using artificial infection methods. Their viral resistance abilities were evaluated based on the method of Herbers et al. (1996).

The number of SMV copies in transgenic plants was analyzed by real time qRT-PCR (Real time Quantitative Reverse Transcription PCR) by using LightCycler ${ }^{\circledR}$ FastStart DNA MasterPLUS SYBR Green I (Roche) and the primers SMVqF and SMVqR (Table 1). The thermal cycle was as follows: denaturation at $95^{\circ} \mathrm{C}$ for $10 \mathrm{~min}$; amplification and binding for 45 cycles $\left(95^{\circ} \mathrm{C}\right.$ for $10 \mathrm{~s}, 58^{\circ} \mathrm{C}$ for $10 \mathrm{~s}, 72^{\circ} \mathrm{C}$ for $20 \mathrm{~s}$ ); and analysis of the flow temperature when the temperature increases from $65-95^{\circ} \mathrm{C}$ for $1 \mathrm{~min}$, continuing to collect fluorescent signals. Temperature $\Delta=20^{\circ} \mathrm{C} \mathrm{sec}^{-1}$. We calculated and determined expression ( $R$ ) by using the $R=2^{-\Delta \Delta c t}$ method of Livak and Schmittgen (2001).

\section{RESULTS AND DISCUSSION}

\section{Transferring the RNAi construct [pK7GW-CPi (SMV-BYMV)] into the tobacco plant}

To improve SMV and BYMV resistance in tobacco plants, the CPi (SMV-BYMV) fragment consisting of conserved regions originating from both SMV and BYMV CP genes was cloned into the vector pK7GWIWG2 (Karimi et al. 2002) in sense- and antisense-orientation. This RNAi-based construct, called pK7GW-CPi (SMV-BYMV) (Lo et al. 2014) (Figure 1), could be expressed as a hairpin RNA structure to trigger the RNAi mechanism once virus infection occurred.

Table 1. A list of oligonucleotide primers used in the study

\begin{tabular}{lc}
\hline Primers & DNA Sequence (5' $\mathbf{- 3}$ ') \\
\hline SMV-CPi-Fi & CACCGCAGCAGAAGCTTACA \\
BYMV-CPi-Ri & ATGTTCCGAACCCCAAGCAA \\
SMVqF & GCCTAGATATGGACTACTGAGGA \\
SMVqR & ATTCACATCCCTTGCAGTGT \\
\hline
\end{tabular}

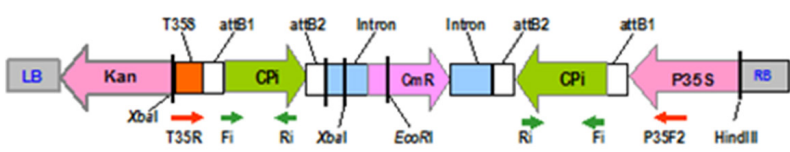

Figure 1. Diagram of pK7GW-CPi (SMV-BYMV). P35S: Promoter CaMV35S; attB1 and attB2: Recombinant positions for $L B$ reaction; LB: left T-DNA border; RB: right T-DNA border; T35S: terminator 35S; Kan: kanamycin resistant; CmR: chloramphenicol resistant; CPi: CPi (SMV-BYMV) incorporation position; Xbal, EcoRI, HindIII: restriction sites; T35R, P35SF2, Fi, Ri: primers SMV-CPi-Fi/BYMVCPi-Ri binding sites. 
The pK7GW-CPi (SMV-BYMV) construct was transferred to A. tumefaciens. Agrobacterium-mediated transformation of this construct via leaf infection and regeneration of tobacco plants were performed as previously described by Topping (1998). Pieces of tobacco leaves were incubated with $A$. tumefaciens for 2 days in the dark, and then washed with antibiotics and transferred to shooting medium supplemented with antibiotics and BAP. After 2-3 weeks, the newly developed shoots appeared. Small buds were removed from the leaf explants and transferred to a fresh medium for rapid bud development. After 1-2 weeks, the shoots were transferred to a rooting medium for root development. The tobacco plants with developed roots growing in the antibiotic medium were transferred into pots.

The CPi (SMV-BYMV) structure was transferred to the tobacco leaf explants twice, using 30 leaf explants each time. The results presented in Table 2 show that from 60 initial explants, 38 survived and generated 82 shoots and 131 plants in vitro. Forty plants were transferred to pots and 26 developed properly. As a control, 30 leaf explants without the transgene were regenerated in a medium with or without antibiotics (Control-0 and Control-1, respectively). As expected, none of the Control-0 explants survived, whereas from 30 Control-1 explants, 105 plants were generated, and 10 were transferred to pots in a greenhouse.

The transgenic tobacco plants were grown in the greenhouse for 3 to 4 weeks, then their leaves were collected to check for the transgene presence by using the specific primers SMV-CPi-F and BYMV-CPi-R. The PCR results show specific bands of approximately 573 bp in size in all 26 lines (Figure 2), suggesting that the CPi (SMV-BYMV) structure has been successfully transferred into C9-1 tobacco plants.

\section{Evaluation of BYMV and SMV resistance of the transgenic tobacco lines under artificial infection conditions}

To test the virus resistance ability of transgenic tobacco plants, 26 transgenic tobacco lines and 10 controls were infected with SMV and BYMV three times at 15-day intervals. After each infection, virus development in the tobacco lines was observed. Figure 3 shows leaf morphology of transgenic and WT plants after infection. Most transgenic plants showed complete resistance, reflected in their normal leaf morphology and full growth (Figure 3A). In contrast, after being infected, all 10 control plants showed severe infection, with mosaic curly leaves and poor growth (Figure 3B). Virus resistance results from 26 transgenic tobacco lines and 10 control plants are presented in Table 3. The statistics from Table 3 show a high percentage of complete resistance of transgenic tobacco lines (19 resistant out of 26 infected, corresponding to $73.08 \%)$.

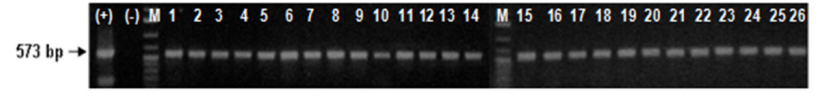

Figure 2. Presence of transgene in transgenic tobacco plants. $(+)$ positive control pK7GW-CPi (SMV-BYMV) plasmid; (-) nontransgenic tobacco plants; M: Marker $1 \mathrm{~kb}$ (Thermo Scientific); 1-26: 26 transgenic tobacco lines.

Table 2. Results of CPi (SMV-BYMV) transformation and tobacco plant regeneration

\begin{tabular}{|c|c|c|c|c|c|c|}
\hline $\begin{array}{l}\text { RNAi structure and } \\
\text { controls }\end{array}$ & $\begin{array}{c}\text { Transformed } \\
\text { leaves }\end{array}$ & Surviving leaves & $\begin{array}{c}\text { Shoots gener- } \\
\text { ated }\end{array}$ & Surviving plants & $\begin{array}{l}\text { Plants trans- } \\
\text { ferred to pots }\end{array}$ & $\begin{array}{c}\text { Transferred to } \\
\text { greenhouse }\end{array}$ \\
\hline pK7GW-CPi (SMV-BYMV) & $2 \times 30=60$ & 38 & 82 & 131 & 40 & 26 \\
\hline${ }^{*}$ Control-0 & 30 & 0 & - & - & - & - \\
\hline${ }^{*}$ Control-1 & 30 & 30 & 79 & 105 & 10 & 10 \\
\hline
\end{tabular}

"Control-0: negative control tobacco plants in the medium with antibiotics. ${ }^{*}$ Control-1: tobacco plants with no transgene in the medium without antibiotics.

Table 3. Artificial infection and resistance ability to SMV and BYMV in transgenic and control plants

\begin{tabular}{|c|c|c|c|c|c|c|c|c|}
\hline \multirow{2}{*}{$\begin{array}{l}\text { Infection } \\
\text { times }\end{array}$} & \multicolumn{4}{|c|}{ Transgenic plants } & \multicolumn{4}{|c|}{ Control plants } \\
\hline & Infected & $\begin{array}{l}\text { Complete } \\
\text { resistance }\end{array}$ & $\begin{array}{c}\text { No resis- } \\
\text { tance }\end{array}$ & $\begin{array}{l}\text { Resistance } \\
\text { percentage }\end{array}$ & Infected & $\begin{array}{l}\text { Complete } \\
\text { resistance }\end{array}$ & $\begin{array}{c}\text { No resis- } \\
\text { tance }\end{array}$ & $\begin{array}{l}\text { Resistance } \\
\text { percentage }\end{array}$ \\
\hline 1 & 26 & 22 & $4++$ & 84.62 & 10 & 3 & $7+++$ & 30.33 \\
\hline 2 & 22 & 22 & 0 & 100 & 3 & 0 & $3+++$ & 0 \\
\hline 3 & 22 & 19 & $3+$ & 86.36 & 0 & 0 & & \\
\hline Total & 26 & 19 & 7 & 73.08 & 10 & & 10 & 0 \\
\hline
\end{tabular}

,,$++++++=$ mild, medium, and severe symptoms, respectively. 
The analytic result from viral quantitative assays by real-time RT-PCR

The number of SMV copies from the transgenic tobacco plant structure was checked by using qRT- PCR. The leaves of three completely resistant lines, called $\mathrm{T}_{0} 5$ - resistant, $\mathrm{T}_{0} 12$ - resistant, and $\mathrm{T}_{0} 19$ - resistant (Figure $3 \mathrm{~A}$ ), and three infected plants (wild type-WT), called WT1- infected, WT2 - infected, and WT3 - infected, from the artificial infection were used as materials for the reaction (Figure 3B).

Total RNA was extracted from $1 \mathrm{~g}$ of tobacco leaves, and $1 \mu \mathrm{g}$ RNA was used for CDNA synthesis. The reaction curve was constructed from three reactions in which E. coli carrying the recombinant $\mathrm{pBT}-\mathrm{SMV}$ was counted using a counting chamber. The DNA concentrations from 3 standard samples were 1000,100 , and 10 copies of DNA $\mu L^{-1}$ respectively (1 cell corresponding to 1 copy). The standard curve equation was constructed using results from the qRT$P C R$ reaction with primers $S M V q F$ and $S M V q R$; the linear curve to determine the number of DNA copies from the threshold cycle was: $Y=-168.15 X+4202.7$, in which $Y$ is the number of copies and $X$ is the threshold cycle values, and $R^{2}=0.898$ ( $R^{2}$ : squared correlation coefficient).

The number of threshold cycles to detect the number of SMV and SMV copies per $1 \mathrm{~g}$ of tobacco leaves are presented in Table 4. Table 4 shows that SMV viruses were found in all samples from transgenic and negative control lines after artificial virus infection. In the WT plants, there were 810 SMV copies on average, which is 1.94 times higher than the transgenic plants (average 416.66 copies). This result suggests that the pK7GW-CPi (SMV-BYMV) construct is activated when SMV infected, inhibiting SMV via the RNAi mechanism; thus, fewer SMV copies are found in these plants. However, in the transgenic plants, the number of copies of SMV is different (Table 4), demonstrating the different levels of disease in different transgenic lines. The qRT-PCR results show that

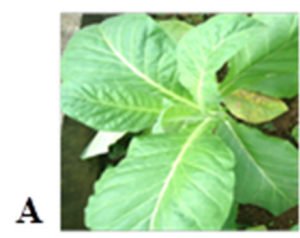

$\mathrm{T}_{0} 5$

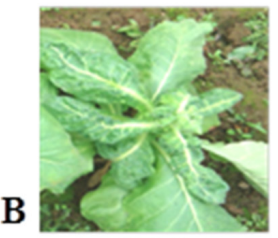

WT1

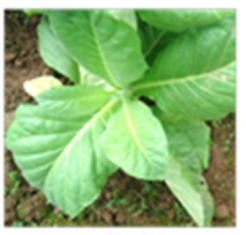

$T_{0} 12$

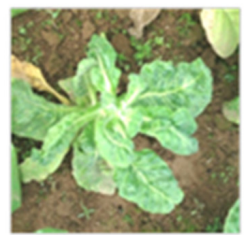

WT2

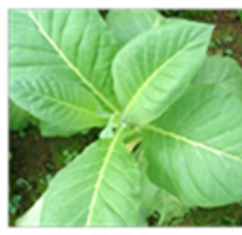

$\mathrm{T}_{0} 19$

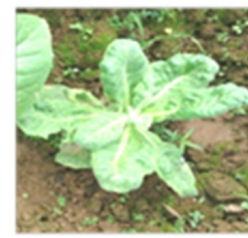

WT3
Figure 3. Leaf morphology of transgenic tobacco and WT plants after being infected three times with SMV and BYMV. A- The transgenic lines $T_{0}-5, T_{0}-12$, and $T_{0}-19$ are completely resistant; B- very severe infection in WT plants. WT1: WT1- infected, WT2: WT2 - infected, and WT3: WT3 - infected.

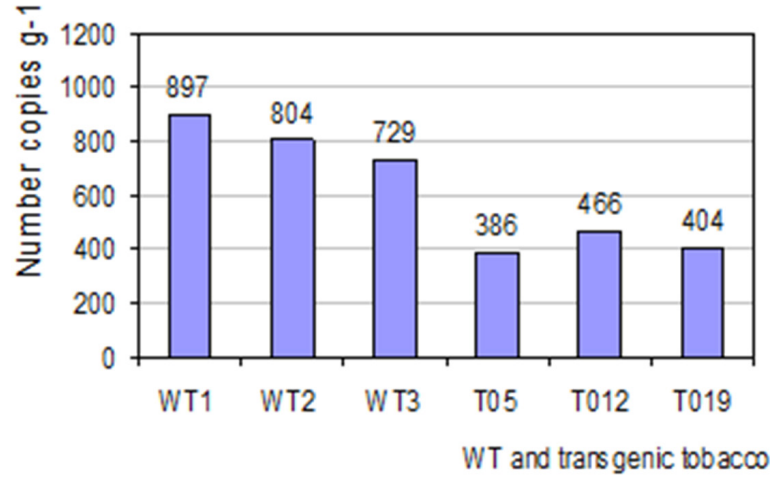

Figure 4. Comparative chart of the number of copies $\mathrm{g}^{-1}$ of tobacco leaf of transgenic tobacco lines and the WT plants after being infected 3 times with SMV and BYMV. WT1, WT2, WT3: the WT plants; $T_{0}-5, T_{0}-12, T_{0}-19$ : the transgenic tobacco lines.

Table 4. Threshold cycle values for detecting SMV and the number of copies $\mathrm{g}^{-1}$ of tobacco leaf

\begin{tabular}{lcc}
\hline Samples & Threshold cycle values & No. of copies $\mathbf{~ g}^{-1}$ of leaf \\
\hline (-) & 28.68 & - \\
WT1 - infected & 19.66 & 897 \\
WT2 - infected & 20.21 & 804 \\
WT3 - infected & 20.66 & 729 \\
T $_{0}$ - resistant & 22.7 & 386 \\
T $_{0} 12$ - resistant & 22.22 & 466 \\
T $_{0} 19$ - resistant & 22.59 & 404 \\
$1 x$ & 19.52 & 1000 \\
$10 x$ & 23.21 & 100 \\
$100 x$ & 25.65 & 10 \\
\hline
\end{tabular}

(-): negative control is $\mathrm{H}_{2} \mathrm{O}$, does not contain cDNA; WT1-infected, WT2-infected, and WT3-infected: three infected wild type plants; $\mathrm{T}_{0}$ 5-resistant, $\mathrm{T}_{0} 12-$ resistant, and $\mathrm{T}_{0}$ 19-resistant: three resistant transgenic plants. 
the expressed RNAi structure CPi (SMV-BYMV) improved SMV resistance in transgenic tobacco lines.

SMV and BYMV belong to the Potyvirus genus, the largest group of plant viruses (Dougherty and Carrington 1988). Their genomes consist of one single-stranded, linear, positive-sense RNA molecule (Dougherty and Carrington 1988). Mosaic disease caused by SMV and BYMV is one of the most common diseases in soybean, which severely reduces soybean yield and quality (Hartman et al. 1999). RNAi is considered to be a modern technique that can effectively protect plants against viruses. The effectiveness of RNAi in generating transgenic plants resistant to viruses has been demonstrated in a number of studies (Yan et al. 2007, Qiong et al. 2011, Pradeep et al. 2012, Zhang et al. 2012). In Vietnam, the first successful RNAi application in transgenic plants was the generation of transgenic tobacco plants resistant to Cucumber mosaic virus (CMV), to Tobacco mosaic virus (TMV), and to both of the viruses at the same time (Pham et al. 2008).

The approach of creating the RNAi structure from the viral CP gene was mentioned by Furutani et al. (2007) as an effective method to improve SMV resistance in soybean. However, in reality, soybean plants can be infected with multiple viruses simultaneously; thus, it is important to construct a transgene that can prevent both viruses causing mosaic disease. Based on the conserved regions of the CP coding sequences of SMV and BYMV, our team has developed an RNAi-based plant expression vector in order to suppress SMV and BYMV infection (Lo et al. 2014). In our study, using RNAi methods, we have achieved very promising results with $73.08 \%$ of transgenic plants showed complete resistance to SMV and BYMV. The analytic result of viral quantitative assays by real-time RT-PCR showed that the number of virus copies in transgenic tobacco plants was reduced on average by more than $51 \%$ compared to the control plants (Figure 4). The results obtained from this study reinforce the environmental-friendly approach to making virus-resistant crops. It also provides an important basis for transferring the CPi (SMV-BYMV) construct to soybean plants to generate soybean plants resistant to SMV and BYMV. Further studies include examining the effect of this method in generating soybean plants resistant to the SMV and BYMV viruses, as well as evaluating inheritance of this transgene in following generations in order to obtain a stable transgenic soybean line that is highly resistant to SMV and BYMV.

\section{CONCLUSIONS}

We have successfully generated new transgenic tobacco plants carrying RNAi [CPi (SMV-BYMV)] resistance to the two types of viruses and $73.08 \%$ of transgenic tobacco lines were fully resistant to SMV and BYMV. By using RT-PCR we have identified the number of virus copies in transgenic tobacco plants, which was reduced on average by more than $51 \%$ compared to the control plants. This promising result shows the potential of transferring the CPi (SMV-BYMV) structure to soybean, to increase resistance of soybean to SMV and BYMV and advance the aims of antiviral soybean breeding in Vietnam.

\section{ACKNOWLEDGMENTS}

The authors would like to express their gratefulness for the help of the Key Laboratory of Gene Technology, Institute of Biotechnology, Vietnam Academy of Science and Technology.

\section{REFERENCES}

Asad S, Haris WAA, Bashir A, Zafar Y, Malik KA, Malik NN and Lichtenstein CP (2003) Transgenic tobacco expressing geminiviral RNAs are resistant to the serious viral pathogen causing cotton leaf curl disease. Archives of Virology 148: 2341-2352.

Baulcombe D (2004) RNA silencing in plants. Nature 431: 356-363.

Bonfim K, Faria JC, Nogueira EO, Mendes EA and Aragão FJ (2007) RNAimediated resistance to bean golden mosaic virus in genetically engineered common bean (Phaseolus vulgaris). Molecular PlantMicrobe Interactions 20: 717-726.

Dougherty WG and Carrington JC (1988) Expression and function of potyviral gene products. Annual Review of Phytopathology 26: 123-143
Furutani N, Yamagishi N, Hidaka S, Shizukawa Y, Kanematsu S and Kosaka Y (2007) Soybean mosaic virus resistance in transgenic soybean caused by post-transcriptional gene silencing. Breeding Science 57: 123-128.

Hartman GL, Sinclair JB and Rupe JC (1999) Compendium of soybean diseases. $4^{\text {th }}$ edn, The American Phytopathological Society Press, St. Paul, 128p.

Herbers K, Meuwly P, Frommer WB, Metraux JP and Sonnewald U (1996) Systemic acquired resistance mediated by the ectopic expression of invertase: Possible hexose sensing in the secretory pathway. Plant Cell 8: 793-803.

Karimi M, Inzé D and Depicker A (2002) GATEWAY ${ }^{\mathrm{TM}}$ vectors for Agrobacterium-mediated plant transformation. Trends Plant Science 7: 193-195. 
Kasai M and Kanazawa A (2012) RNA silencing as a tool to uncover gene function and engineer novel traits in soybean. Breeding Science 61: 468-479.

Livak KJ and Schmittgen TD (2001) Analysis of relative gene expression data using real-time quantitative PCR and the 2(-Delta Delta $C(T)$ ) method. Methods 25: 402-408.

Lo TMT, Le VS, Chu HH and Chu HM (2014) Development of RNAiBased vector aims at creating antiviral soybean plants in Vietnam. International Journal of Bioscience, Biochemistry and Bioinformatics 4: 208-211.

Pham TV, Nguyen VB, Le VS, Chu HH and Le TB (2008) Create tobacco plants resistant to cucumber mosaic virus disease by RNAi. Vietnamese Journal of Biotechnology 6: 679-687.

Pradeep K, Satya VK, Selvapriya M, Vijayasamundeeswari A, Ladhalakshmi D, Paranidharan V, Rabindran R, Samiyappan R, Balasubramanian P and Velazhahan R (2012) Engineering resistance against Tobacco streak virus (TSV) in sunflower and tobacco using RNA interference. Biologia Plantarum 56: 735-741.
Qiong H, Yanbing N, Kai Z, Yong L and Xueping Z (2011) Virus-derived transgenes expressing hairpin RNA give immunity to Tobacco mosaic virus and Cucumber mosaic virus. Virology Journal 8: 41.

Saghai MMA, Soliman KM, Jorgensen RA and Allard RW (1984) Ribosomal DNA spacer - length polymorphisms in barley: Mendelian inheritance, chromosomal location and population dymnamics. Proceedings National Academic Science USA 81: 8014-8018.

Topping JF (1998) Tobacco transformation. Methods of Molecular Biology 81: $365-372$.

Yan PQ, Bai XQ, Wan XQ, Guo ZK, Li LJ, Gong HY and Chu CC (2007) Expression of TMV coat protein gene RNAi in transgenic tobacco plants confer immunity to tobacco mosaic virus infection. Yi Chuan 29: 1018-1022.

Zhang C, Song Y, Jiang F, Li G, Jiang Y, Zhu C and Wen FF (2012) Virus resistance obtained in transgenic tobacco and rice by RNA interference using promoters with distinct activity. Biologia Plantarum 56: 742-748. 